\title{
Electrochemical Characterization of Regularly-aligned Nanopore Array Membranes Filled with Electrolyte Solutions and their use for Detection of Nucleic Acid Hybridization
}

\author{
J. S. Ellis ${ }^{\mathrm{a} *}$, G. Herzog ${ }^{\mathrm{a}}$, B. Glynn ${ }^{\mathrm{b}}$, and D. W. M. Arrigan ${ }^{\mathrm{c}}$ \\ ${ }^{a}$ Life Sciences Interface Group, Tyndall National Institute, University College Cork, \\ Ireland \\ b Molecular Diagnostics Research Group, National Centre for Biomedical Engineering \\ Science, National University of Ireland Galway, Ireland \\ c Nanochemistry Research Institute, Department of Chemistry, Curtin University, Perth, \\ Australia \\ * present address: Dept. of Mechanical \& Industrial Engineering, University of Toronto, 5 King's College \\ Rd., Toronto, ON M5S 3G8, Canada
}

\begin{abstract}
We report on the electrochemical characterization of regularlyaligned cylindrical nanopore arrays supported in silicon nitride membranes and preliminary results for the detection of nucleic acid hybridization on the nanopore walls. A range of nanopore arrays with diameters between 40 and $150 \mathrm{~nm}$ were examined. We tested the effect of pore diameter, number of pores, electrolyte concentration and surface chemistry on the conductance of the nanopore membranes. The pores were functionalized with singlestranded DNA and conductance measurements were performed before and after hybridization. In many cases, changes in current rectification were observed following hybridization, which is discussed as a strategy for nucleic acid hybridization and interactions.
\end{abstract}

\section{Introduction}

Nanopore electrochemistry has received significant attention in recent years as a technique for the detection of biochemical interactions (1). Measurements of biochemical events in nano-scale confinement offer a number of advantages over conventional bulk techniques. These include i) faster response times and shorter diffusion lengths due to the increased surface-to-volume ratios, ii) improved control of molecular transport through manipulation of the electrical double layer, iii) a move towards single-molecule biosensing, and iv) the ability to probe interactions on biologically relevant length scales. Additionally, recent advances in micro- and nano-fabrication techniques have improved the reproducibility of making such nano-scale detection systems.

Electrochemical measurements are performed within nano-scale channels, typically supported within thin membranes. These can include single channels or arrays of channels. In the typical setup, the channel is filled with an electrolyte solution and a potential is applied across the membrane. Geometric and surface properties of the channels can be determined from conductance measurements, and a variety of techniques can be used to detect biochemical interactions within the pores. 
To explore the use of arrayed nanopore membranes in biosensing, we present preliminary results for i) the characterization of nanopore array membranes using conductance measurements, and ii) electrochemical detection of nucleic acid hybridization at functionalized pore walls. Conductance measurements were performed on nanopore membranes in potassium chloride electrolyte over a broad range of ionic strengths. A variety of pore diameters, array sizes and surface treatments are tested, including biochemical functionalization with single-stranded DNA (ssDNA). Then, the effects of solution-based nucleic acids on the conductance of the nanopore membranes were measured for both untreated and nucleic acid-functionalized nanopores. In certain cases, current rectification behavior was observed, which is characterized by nonlinearity of the current-potential curve. Rectification has been attributed to asymmetric geometry and charge distributions on the device surface $(2,3)$, and has been proposed as a method to detect biochemical interactions on the pore wall (4). In cases where hybridization was possible, conductance measurements were performed throughout the hybridization process. We show evidence of nucleic acid hybridization detection within arrayed nanopores, whereby different rectification behavior is seen for complementary versus non-complementary DNA. Scanning-electron microscopy were performed to verify the geometric properties of the nanopore arrays and fluorescence microscopy was used validate nucleic acid hybridization.

The paper begins with a review of nanopore electrochemistry and nano-scale detection of nucleic acids. Then, the experimental methodology is presented, followed by the preliminary results for the characterization of the nanopore arrays. A treatment of the hybridization results follows, as well as a brief discussion of the rectification behavior as possible evidence for hybridization.

\section{Background}

\section{Conductance Theory.}

Conductance measurements can be applied to probe the geometric and surface charge parameters of nanopore and nanopore array membranes. In the typical setup, the nanopore membrane separates two reservoirs filled with an electrolyte solution. Potassium chloride at various concentrations is used, since the mobilities of both ions are nearly equal $\left(\mu_{\mathrm{K}+}=7.6 \times 10^{-8} \mathrm{~m}^{2} \cdot \mathrm{V}^{-1} \cdot \mathrm{s}^{-1}\right.$ and $\left.\mu_{\mathrm{Cl}-}=7.9 \times 10^{-8} \mathrm{~m}^{2} \cdot \mathrm{V}^{-1} \cdot \mathrm{s}^{-1}\right)$ (5). The ionic conductance is the ratio of the ionic current to the applied potential, and is a combination of the bulk solution conductance and the pore conductance. The bulk conductivity is a function of the solution concentration and composition. The conductance of an array of nanopores is governed by a number of factors, including the pore geometry, the electrolyte properties and concentration, the ionic mobilities, and the surface charge within the pore $(2,6,7)$. The conductance $G$ depends on two effects: (i) the bulk effect is affected by the bulk conductivity of the pore and the electrolyte solution, and (ii) the exclusion effect, important at low electrolyte concentrations, is governed by the surface charge along the pore wall. In the high concentration, low surface charge regime, the bulk effect dominates and $G$ is given by: 


$$
\text { I } ? \frac{C_{\text {ratg }}}{N_{\text {r gtg }}}\left[H\left(E_{-}-E_{-}\right) E_{d w m}\right]
$$

where $A, L$, and $r$ are the cross-sectional area, length, and radius of the pore, $F$ and $\mu$ are the Faraday constant and the ionic mobilities, and $C_{\text {bulk }}$ is the bulk concentration. At low ionic strengths, the conductance is very sensitive to surface charge effects, so the conductance becomes

$$
I ? \frac{C_{\text {ratg }}}{N_{\text {ratg }}}\left[H\left(E_{-}-E_{-}\right) E_{d u m}-\frac{4 E_{-}^{s}}{t_{r q t g}}\right]
$$

where $\sigma$ is the surface conductance on the pore wall (8-10). This fact can be exploited for biosensing applications within nanopores by manipulating the pore wall chemistry.

In some cases, the current-potential curve used to measure conductance across nanopores is not linear. This phenomenon, known as current rectification, could be due to variety of factors, most notably asymmetric charge distributions along the walls of the pore and asymmetric pore geometry, particularly conically shaped pores. In essence, the current magnitude at the negative end of the potential sweep is different than that for the same potential on the positive side of the sweep. The effect is due to the difference in resistance for ions entering at one end of the pore compared to the other end, either due to geometric or surface charge differences. Such behavior has been compared to an electrical diode (4), and changes in current rectification have been used as a detection mechanism for surface-bound biochemical interactions $(4,11)$.

\section{$\underline{\text { Nano-scale Detection of Nucleic Acids }}$}

Techniques that have been applied to detect biomolecules and measure biomolecular interactions within nanopore devices include impedance measurements $(12,13)$, the resistive pulse technique $(7,8,14-19)$, and current rectification behavior $(4,11)$. Vlassiouk et al. (12) used impedance measurements to detect DNA hybridization in pores with different entrance and exit radii (20 and $200 \mathrm{~nm}$ ). They found an increase in the absolute impedance on hybridization, due to blocking of pores, when the 20-nm side was in contact with the working electrode. Wang and Smirnov (13) fitted AC impedance measurements of an array of pores in an anodized alumina membrane, to a fourcomponent equivalent circuit and extracted pore conductance and capacitances. There was little difference between the absolute impedances at high concentrations, but the authors found an increase in the conductance at low ionic strengths for pores with immobilized ssDNA, compared to unmodified pores.

The most common method for detection of biomolecules using nanopore devices is the so-called resistive-pulse, or Coulter, technique $(7,8,14-19)$, which has also been treated theoretically (20-22). In this method, a potential is applied across the membrane such that DNA added to one side of the pore will electromigrate through the pore. When the DNA enters the pore, it blocks a portion of the pore area, thereby reducing the radius to electrolyte charge carriers, and decreasing the conductance. This appears as a spike in the current-time plot, where the amplitude and duration of the spike can yield information about the DNA. The amplitude and direction of the current shift can depend on the electrolyte concentration, indicating that the electrostatic charge and the pore blockage 
influence the current and translocation (17). The translocation time and current shift also depend on interactions with the pore walls, and such devices have been shown to detect base-pair mismatches in ssDNA modified pores (15).

While many studies use chronoamperometric detection of the translocation events, we use here current-potential $(I-V)$ curves and conductance measurements, which can be achieved using potentiostats available in most electrochemistry labs, without requiring more expensive high-speed current measurements. In the presence of current rectification, nucleic acid detection is possible and, with further development, may even be used for the measurement of real-time nucleic acid hybridization.

\section{Materials and Methods}

\section{$\underline{\text { Materials }}$}

Fabrication. Nanopore arrays supported in silicon nitride membranes were produced as described previously (23). In brief, a $525-\mu \mathrm{m}\langle 100\rangle$ silicon wafer was coated thermally with a pad oxide layer, followed by low pressure chemical vapor deposition of a 100-nm thick nitride layer. The nanopore arrays were patterned using electron-beam lithography onto an E-beam resist on the top side of the $\mathrm{Si}_{3} \mathrm{~N}_{4}$. The nanopores were formed in the $\mathrm{Si}_{3} \mathrm{~N}_{4}$ using a magnetic zero-resonant induction etch, a form of inductively coupled plasma etch employing fluorocarbon gas chemistry $\left(\mathrm{CHF}_{3} / \mathrm{CH}_{2} \mathrm{~F}_{2} / \mathrm{Ar}\right)$. The back-side nitride layer was patterned using photolithography, then the nitride, pad-oxide and bulk silicon were removed using a PERIE nitride-on-oxide etch, 10:1 HF wet-etch, and $\mathrm{KOH}$ wet-etch. Prior to the HF etch, the nanopore surface on the top-side was spin-coated with ProTEK B3 polymer film (Brewer Science, Montana, USA) to protect the $\mathrm{Si}_{3} \mathrm{~N}_{4}$ membrane during the final fabrication and dicing steps. This layer was removed after dicing by immersion in ProTEK Remover 100 solution. All processing was carried out within the Central Fabrication Facility at Tyndall National Institute. The final chips featured a $100-\mathrm{nm}$ thick, $500 \mu \mathrm{m} \times 500 \mu \mathrm{m} \mathrm{Si}_{3} \mathrm{~N}_{4}$ membrane supported by silicon, with the nanopore arrays were centered in the $\mathrm{Si}_{3} \mathrm{~N}_{4}$ membrane. Nanopores with diameters of $30,40,50,100$, and $150 \mathrm{~nm}$ were produced, with pore center-center separation distance of $10 d$ in a hexagonal array, where $d$ is the individual pore diameter. All chips were cleaned in $\mathrm{O}_{2}$ plasma (March Plasmod, California, USA) prior to experimental characterization. A sample of nanopore membranes were SEM imaged using a Nova 630 NanoSEM (FEI, Eindhoven, The Netherlands) with the in-lens detector with immersion mode of operation (HRSEM mode) to determine the verify geometric properties of the nanopore arrays.

Chemicals. All chemicals were of analytical grade or better, and were used as received, unless otherwise indicated. Potassium chloride powder, 1,4-phenylene diisothiocyanate (PDITC;98\%), 1M Tris-HCl pH 7.4 buffer, acetone, isopropyl alcohol, methanol, hydrochloric acid (37\%), sulfuric acid (98\%), sodium dodecyl sulfate (SDS;99\%), and saline sodium citrate (SSC; 20X) were obtained from Sigma-Aldrich Ireland, Ltd, and diluted as required with purified water (18.2 M $\Omega$; Elga Purelab Ultra, Veolia Water Systems, Ireland). The same water was used for rinsing. 3aminopropyltriethoxysilane (APTES) was obtained from either Fluorochem, Ltd. (UK) or 
Sigma-Aldrich Ireland, Ltd. Single-strand oligonucleotides were obtained from Eurofins MWG Operon (Ebersberg, Germany). Capture probes were modified with an amino group on the $5^{\prime}$ end. Reporter probes were either unmodified, or modified with a CY5 fluorescent tag on the $5^{\prime}$ end, for verification of the hybridization. The ssDNA molecules used are shown in Table I. Unless otherwise indicated, the concentration of the ssDNA used was $100 \mathrm{nM}$.

TABLE I. Capture and reporter probe sequences used in the study

\begin{tabular}{cc}
\hline Capture Probe & Sequence $\mathbf{( 5}^{\prime}$-3') \\
\hline Cap1 & TTT TAA CAG CCC CTC GAC AC \\
Cap2 & GTC TAG AAA CTG CGA GTC TAT C \\
Cap3 & AAC TGC GAG TCT ATC AAT CTC T \\
Rep1 & GTG TCG AGG GGC TGT TAA AA \\
Rep2 & GAT AGA CTC GCA GTT TCT AGA C \\
Rep3 & AGA GAT TGA TAG ACT CGC AGT T \\
\hline
\end{tabular}

Complementary Streptococcus pneumoniae tmRNA molecules (240bp) were synthesized in-house using Nucleic Acid Sequence Based Amplification (NASBA; bioMérieux, Marcy l'Etoile, France). The NASBA process was performed using $1 \mathrm{ng} S$. pneumoniae total RNA as a template. The protocol was modified to generate sense strand tmRNA molecules $(24,25)$. Following amplification, tmRNA products were purified using QiaQuick PCR purification kit (Qiagen, West Sussex, UK) and quantified on Agilent 2100 bioanalyser and RNA Nano-Chip (Agilent, Berkshire, UK). The concentration of added tmRNA was $1 \mathrm{ng} / \mu \mathrm{l}$.

\section{Experimental Setup}

Surface Chemistry and Storage. To facilitate filling of the nanopore arrays with $\mathrm{KCl}$ solutions, the chips were silanized with an amino-terminated silane layer (APTES), before insertion in the electrochemical cell. The silanization procedure is based on one described previously (26). The pores were soaked sequentially in a 1:1 mixture of $\mathrm{HCl}$ :methanol, followed by $\mathrm{H}_{2} \mathrm{SO}_{4}$ (32\%), each for 15 minutes. After soaking, the chips were rinsed with and soaked in acetone and isopropyl alcohol for 5 minutes in each, followed by rinsing with deionized water and drying in an oven at $90^{\circ} \mathrm{C}$. Because the membranes are fragile, all rinsing was performed with a dropper, so that the liquid flowed over the chip, instead of impacting it directly.

After drying, the chips were plasma-cleaned for 20 minutes in air (Harrick Plasma, New York, USA), then immersed in a 3\% APTES solution in a 19:1 methanol:deionized water mixture for approximately 2 hours. Following silanization, the chips were rinsed with copious amounts of methanol and deionized water to remove any unbound silane molecules, then cured at $120^{\circ} \mathrm{C}$ for 15 minutes in a fan-operated oven. After curing, each silanized chip was either further functionalized, or assembled into the electrochemical cell.

The chips were functionalized with 1,4-phenylene diisothiocyanate (PDITC) linker chemistry, by immersion in $1 \mathrm{mM}$ PDITC in 10\% pyridine in dimethyl formamide (DMF) for 2 hours, followed by rinsing in DMF and 1,2-dichloroethane and drying in a 
fan-operated oven at $80^{\circ} \mathrm{C}$ for at least 20 minutes. Dilute solutions of the single-stranded oligonucleotides in $20-\mathrm{mM}$ Tris- $\mathrm{HCl}$ buffer were applied to the membranes in $1-2 \mu \mathrm{l}$ spots, and allowed to incubate overnight in a humidity chamber at $37^{\circ} \mathrm{C}$. After immobilization, the chips were rinsed with methanol and purified water, and then dried at $80^{\circ} \mathrm{C}$. The unbound PDITC moieties were capped by immersion of the chips in $50 \mathrm{mM} 6-$ amino-1-hexanol and $150 \mathrm{mM}$ N,N-diisopropylethylamine in DMF for at least 2 hours. Finally, the chips were rinsed sequentially with DMF, methanol, and water and dried at $80^{\circ} \mathrm{C}$.

Following silanization or immobilization, the electrochemical cell was formed by gluing of each chip to one end of a 3-mm internal diameter glass tube. This formed one half of the electrochemical cell. The glued chip-tube apparatus was cured overnight under vacuum. Once set, the nanopore arrays were filled with $1-\mu \mathrm{M} \mathrm{KCl}$ solution, then stored immersed in 1- $\mu \mathrm{M} \mathrm{KCl}$ for 3-4 days before use. They were then used for electrochemical measurements within approximately 2-3 weeks. Nanopore arrays that had not been silanized prior to filling did not yield consistent results. The amino functionalities of the immobilized APTES rendered the make the pore walls more hydrophilic and allowed the pores to be filled with electrolyte solution.

Hybridization Verification. Offline verification of the hybridization of the complementary ssDNA and RNA was performed via fluorescence imaging. First, $5 \mu \mathrm{l}$ of $4 \mu \mathrm{M}$ Cy5-modified complementary DNA in 2x SSC was pipetted onto a Hybri-coverslip (Sigma). The capture probe-modified silicon nitride substrates were placed modified-face down on the coverslips, so the oligo-dilutions flooded the substrate surface. The substrates were incubated overnight in a darkened humidity chamber at $42^{\circ} \mathrm{C}$. Following incubation, the substrates were rinsed sequentially with 2x SSC with $0.1 \%$ SDS (sodium dodecyl sulfate, $1 \mathrm{x}$ SSC with $0.1 \%$ SDS, and $0.1 \mathrm{x}$ SSC with $0.1 \%$ SDS, then rinsed with water, and finally dried at $80^{\circ} \mathrm{C}$ for 20 minutes. The substrates were stored in a darkened chamber under vacuum up to 1 week, and then imaged with an Olympus BX51 microscope with an Olympus DP71 CCD camera fitted with the appropriate filters. The excitation light was supplied from an X-Cite 120PC fluorescent lamp (Lumen Dynamics Group, Mississauga, Canada). Images were captured using Cell-F imaging software (Olympus Soft Imaging Solution GmbH, Germany) on a PC computer.

\section{$\underline{\text { Electrochemical Measurements }}$}

All electrochemical measurements were performed using an Autolab 302N potentiostat (Ecochemie, Utrecht, The Netherlands). NOVA 1.5/1.6 software was used to run the measurements and fit the impedance scans. Conductance measurements were carried out over a range of $\mathrm{KCl}$ concentrations from $10^{-6}$ to $1 \mathrm{M}$. A schematic of the electrochemical cell setup is shown in Figure 1. 


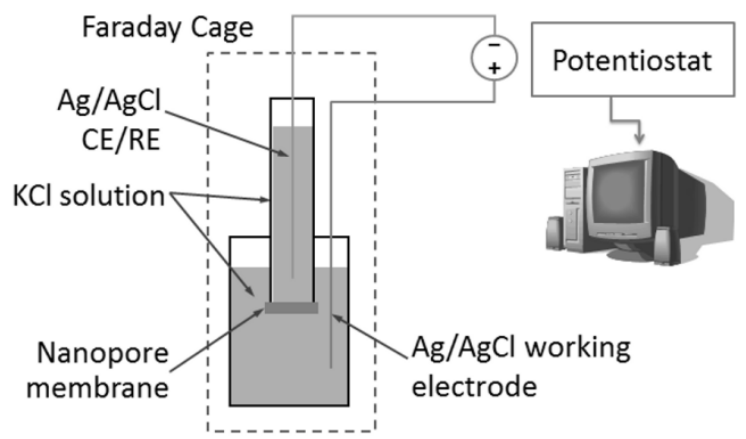

Figure 1. Schematic of the electrochemical experimental setup. The nanopore array membrane is glued to one end of a glass capillary tube and immersed in a glass vial containing the electrolyte solution.

Conductance. The conductance was measured using the linear sweep voltammetry function in NOVA, which was modified to suit the requirements. A run began with 60s rest at $0 \mathrm{~V}$, followed by 60 s equilibration at $300 \mathrm{mV}$. The voltage was then swept from -300 to $+300 \mathrm{mV}$ at $50 \mathrm{~ms} \mathrm{~V}^{-1}$, and the conductance was taken as the slope of the current-voltage line between -100 and $+100 \mathrm{mV}$. For highl y rectified measurements, the slope was determined over a smaller potential window. Conductance measurements were performed over a broad range of $\mathrm{KCl}$ concentrations by sequential additions of standardized $\mathrm{KCl}$ solutions, normally between $1 \mu \mathrm{m}$ and $1 \mathrm{M}$.

Nucleic Acid Detection. To detect the effect of nucleic acid addition on the conductance signal, three strategies were used. In the first, offline overnight hybridization, conductance measurements in $\mathrm{KCl}$ were performed as described above, up to a specific $\mathrm{KCl}$ ionic strength. Then, complementary or non-complementary ssDNA were added to the cell, and allowed to hybridize overnight in a humidity chamber. Following hybridization, the $\mathrm{KCl}$ additions were continued. For the second strategy, nucleic acid (NA) physisorption, concentration additions of $\mathrm{KCl}$, from $1 \mu \mathrm{M}$ to approximately $1 \mathrm{mM}$, were performed to establish the working parameters of the membrane. Then, concentration additions of cDNA were made, from 0.1 to $10 \mu \mathrm{M}$. For the final strategy, online overnight hybridization, the conductance was measured hourly before addition of ssDNA to the functionalized nanopore membrane, and then throughout the overnight hybridization, over 16 hours. Hybridization was measured during this process as the degree of rectification $(4,11)$, which is determined from the $I-V$ curve as the ratio of current at a negative potential, $\mathrm{I}_{-\phi}$, to the current at the same positive potential, $\mathrm{I}_{+\phi}$, so that $r=\left|\mathrm{I}_{-\phi}\right| / \mathrm{I}_{+\phi} \mid$. All overnight hybridization experiments were performed in a humidity chamber.

\section{Results \& Discussion}

Imaging

SEM images of various aspects of the nanopore arrays are shown. Figure 2a shows an entire hexagonal array for a $50-\mathrm{nm}, 390$ pore array. The dimensions of the entire array are 
approximately $8.2 \mu \mathrm{m} \times 9.8 \mu \mathrm{m}$. Pores are shown in detail in Figure $2 \mathrm{~b}$ and c. The pore center-to-center spacing for all arrays used is $10 \times($ pore diameter $)$, as indicated.

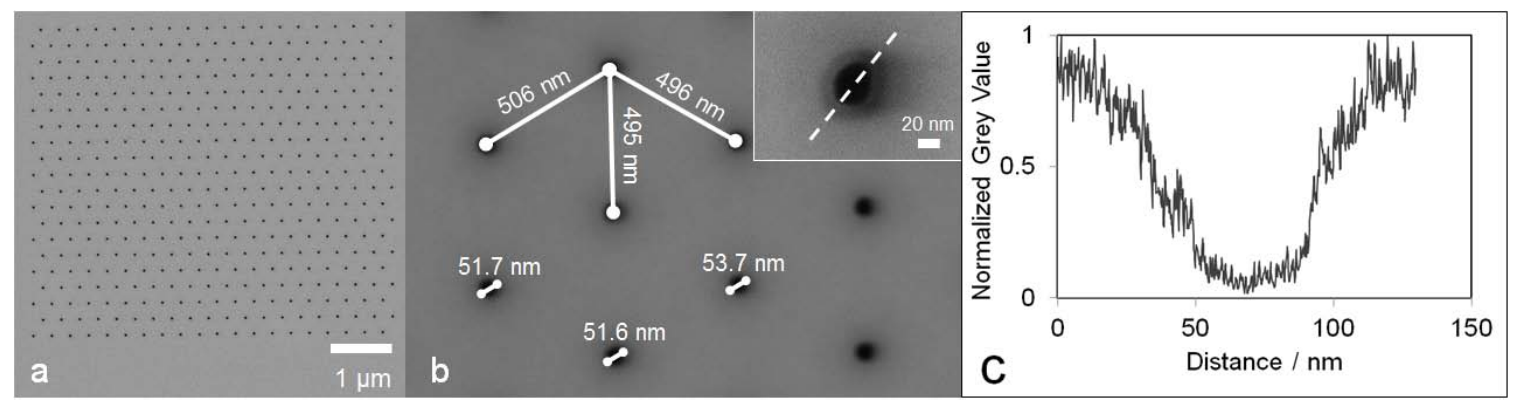

Figure 2. SEM imaging of various nanopore layouts. (a) Full hexagonal 390-pore array of 30-nm diameter pores, (b) detail of a 50-nm diameter pore array, (b-inset) single 50-nm diameter pore from array, (c) normalized grey-scale depth profile of pore shown in (binset) along dashed line. The image acquisition conditions for magnification, acceleration voltage $(\mathrm{kV})$, and working distance $(\mathrm{mm})$ were: (a) [10.2k X, 5.0, 7], (b) [148.7k X, 5.0, 6], (b-inset) [1.2M X, 5.0, 6].

Hybridization Validation.

Fluorescence microscopy was used to verify the binding of complementary oligonucleotides and RNA to the ssDNA functionalized surface. Figure 3 shows fluorescence images of a flat $\mathrm{Si}_{3} \mathrm{~N}_{4}$ surface, functionalized with Cap2 (Table 1), following 1.5 hours of silanization, and hybridization overnight with Rep2 (Figure 3a) and Rep3 (Figure 3b). In all cases, the entire silicon nitride surface was silane treated prior to capture probe immobilization and the capture probes were applied as droplets of approximately 1-2 $\mu \mathrm{l}$. Following immobilization, the entire surface was covered overnight with the reporter probes, and then washed prior to imaging.

The brighter spots indicate the presence of fluorescent adsorption to the surface following wash-off. In the first image (a), the reporter oligonucleotide (Rep2) was complementary to the immobilized capture probe (Cap2), demonstrating that specific adsorption occurred on the silanized and functionalized surface. The second image (b) shows that little adsorption occurred for the non-complementary reporter probe (Rep3), although there was a small amount of non-specific adsorption, as is apparent from the faint spot pattern. Binding of the 240bp complementary RNA (approx. 1\% v/v of NASBA1 concentrate in $20 \mathrm{mM}$ Tris buffer) to a Cap2-modified surface is shown in Figure 3c. This demonstrates specific adsorption of the complementary RNA to the immobilized capture probe.

These images verify i) that the silanization process is indeed modifying the surface, ii) the complementary ssDNA (Rep2) and RNA bind to DNA-functionalized (Cap2) surface, and iii) there is little specific adsorption of the non-complementary DNA (Rep3). In all cases, there was some background fluorescence over the entire surface which was not removed by washing. This could be due to non-specific adsorption of the reporter oligonucleotide to the aminosilane surface. The adsorption and imaging conditions are described above in Materials and Methods. 


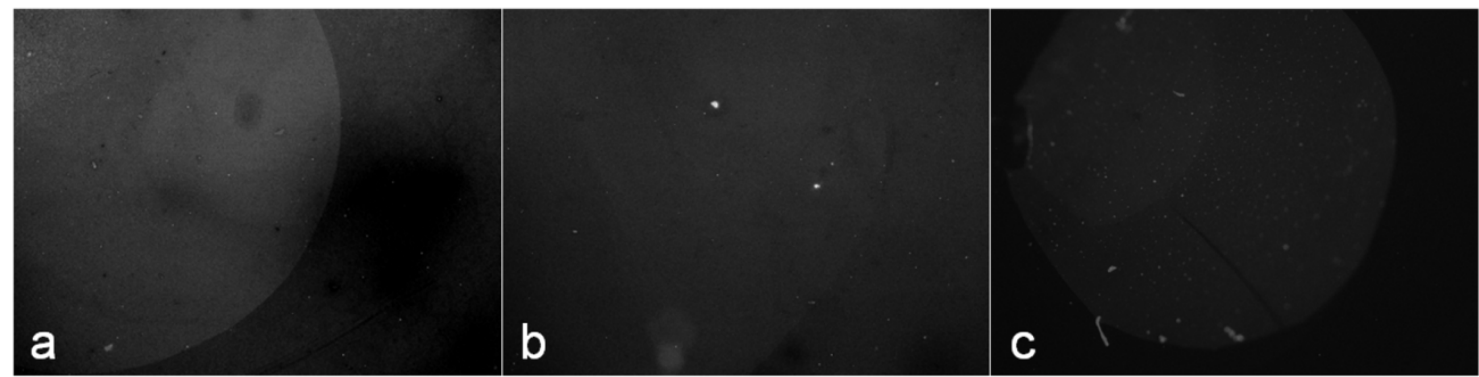

Figure 3. Fluorescence microscopy images of (a) complementary Rep2 oligonucleotide, (b) non-complementary Rep3 oligonucleotide and (c) complementary RNA overnight hybridization, to a Cap 2 ssDNA functionalized surface.

\section{$\underline{\text { Conductance Measurements }}$}

The concentration dependence on the measured conductance was examined over a range of $\mathrm{KCl}$ concentrations from $10^{-6}$ to $1 \mathrm{M}$, for a variety of pore and array geometries. Figure 4 shows two representative plots of linear sweep conductance for 40-nm diameter pores in 390-pore arrays. In both of these runs, the potential sweeps do not go through the origin. This may be due to concentration asymmetry in the cell (c.f. Figure 1).
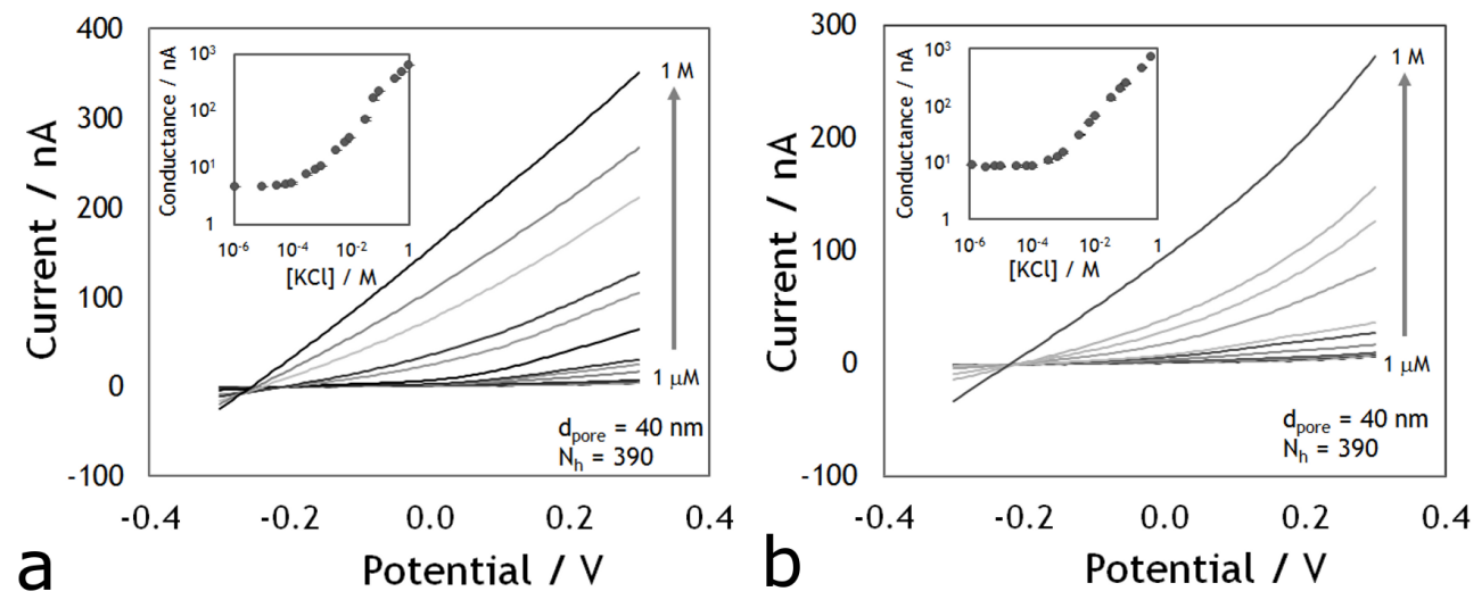

Figure 4. Representative plots of linear sweep conductance measurements over a series of $\mathrm{KCl}$ concentrations $(1 \mu \mathrm{M} \rightarrow 1 \mathrm{M}$ ) for 40 -nm diameter 390-pore arrays, silanized with APTES. Each line is an average of three repetitions at each concentration. (a) and (c) show the $I-V$ plot for one run, and (b) and (d) for another.

Another feature evident in the plots in Figure 4 is the curvature in the currentpotential plot. This is especially evident in Figure 4c, although it is also evident at the lower concentrations in Figure 4a. This feature is known as current rectification, and has been discussed in detail by a number of researchers for experimental studies of nanopores (3,27-31), and has also been considered theoretically (2,32-35). Current rectification has also been proposed as a method for the detection of biochemical interactions $(4,11)$. 
To examine array effects, the influence of ionic strength on conductance was tested on membranes of different array sizes. Figure 5 shows the conductance values over a range of concentrations for two 50-nm pore membranes: a 390-pore array and a single pore. Both membranes were silanized with APTES prior to insertion in the electrochemical cell. The behavior of the single pore matches very well to theory, for a surface charge of $30 \mathrm{mC} \cdot \mathrm{cm}^{-2}$. However, the experimental values with 390 pores are significantly lower than the theoretical results, by approximately an order of magnitude.

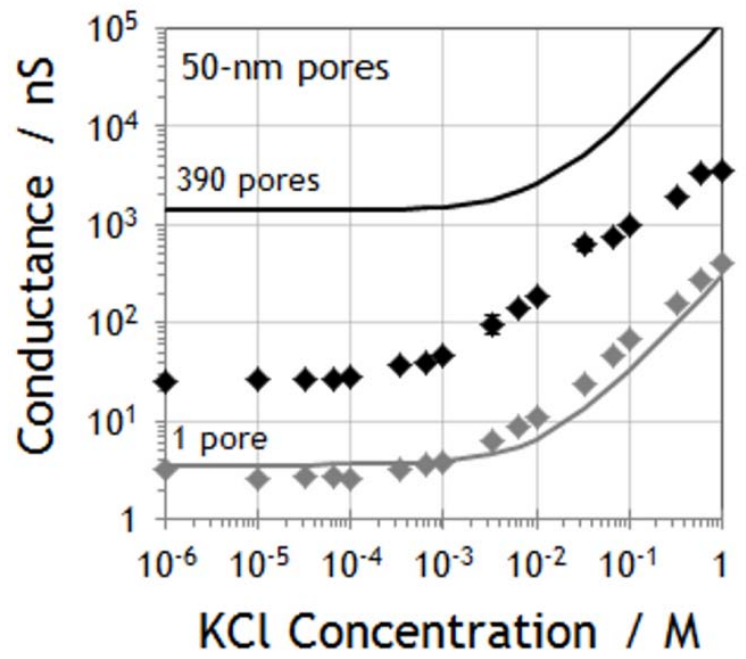

Figure 5. Experimental (data points) and theoretical (lines) results for $50-\mathrm{nm}$ diameter pores for $\mathrm{N}_{\mathrm{h}}=1$ (grey) and $\mathrm{N}_{\mathrm{h}}=390$ pores (black). The theoretical lines are calculated using Eq. 2 with $\sigma=30 \mathrm{mC} \cdot \mathrm{cm}^{-2}$. Error bars show one standard deviation.

The reduced signal for the array of pores, seen in nearly all runs performed with the multiple pores, could be attributed to a variety of factors. One possibility is that some of the pores were not completely formed during the fabrication procedure, and are either not fully formed cylindrical pores, or do not even extend through the entire membrane thickness. Alternatively, the silanization process may have resulted in reduced pore diameters, due to formations of cross-linked multilayers. However, it is unlikely that this effect is entirely responsible for the overestimation, since the decrease in signal would require an average reduction in pore diameter by $72 \%$. SEM images of $500-\mathrm{nm}$ pores indicate that there may be some reduction in pore diameter, but it is only on the order of $20-30 \%$ and would not account for such a strong degree of signal reduction (images not shown). Another possibility, which could be related to the first, is that the pores did not form fully during fabrication, and were instead conical. This was not verified experimentally, although there is initial evidence of conical pores from the grey-scale image depth profile shown in Figure 3c.

\section{Detection of Nucleic Acid Hybridization}

Hybridization was inferred from conductance measurements and rectified currents based on the analysis of three different strategies: i) Offline Overnight Hybridization, ii) Nucleic Acid Physisorption, and iii) Online Overnight Hybridization. The results are described and discussed below. 
Offline Overnight Hybridization. This strategy demonstrated the effect of hybridization on the conductance signal. Results of these measurements are shown in Figure 6, whereby there is some rectification in all cases, even before addition of the complementary nucleic acid. In two cases, there is little change in rectification behavior: for the control (a), C2-R2 (b), there are changes in the slope of the $I-V$ curves, but the rectification remains constant. For the C3-R3 measurement (c), the rectification remained the same generally, but the conductance decreased following overnight hybridization and $\mathrm{KCl}$ additions. It is important to note that the main plots in Figures 6 display only the $I-V$ curves immediately before and after hybridization. This is done to illustrate the rectification behavior following hybridization, since rectification is most evident at moderate concentrations, before bulk effects begin to dominate. A plot of $I$ - $V$ curves over the entire range is qualitatively similar to those shown in Figure 4. The insets in Figure 6 display the calculated conductance values over the full concentration range.
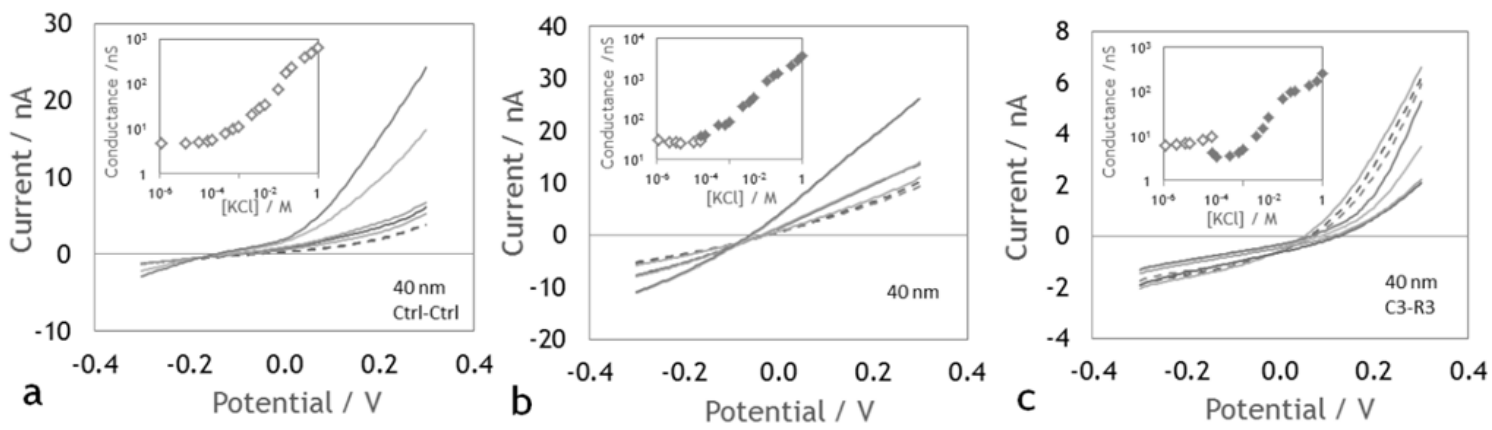

Figure 6. Plots of various offline overnight hybridization experiments for different surface modifications and hybridizing nucleic acids. For each experiment, the top plot shows the averaged $I-V$ curves just before (dashed) and just after (solid) overnight hybridization. $I-V$ curves at higher concentrations are omitted from the plots. The insets show the conductance curves before (open) and after (closed) hybridization. In the insets, the conductance values over the entire concentration range are displayed to demonstrate the curvature. All membranes had 390 pores.

Nucleic Acid Physisorption. The effect of increasing nucleic acid concentrations on the membrane conductance was tested using the NA Physisorption strategy. Results for these experiments are shown in Figure 7. In each case, there is some current rectification present in the system, even before DNA addition. In most cases, during the nucleic acid additions, the degree of rectification did not change, and only the slope of the $I-V$ line was affected. This amounted to an increase in conductance, followed by a subsequent decrease (Figure 7a-b). Since there was no surface functionalization with ssDNA, any changes should be due to bulk effects from the added charges of the DNA and physisorption on the pore walls. As well, in all three cases, the peak in conductance was observed to occur near $1 \mu \mathrm{M}$ ssDNA. It is important to note that in Figure 7a and b, as with Figure 6, only $I-V$ curves just prior to and following addition of the ssDNA are presented. The curves at higher and lower concentrations are omitted to facilitate display of the change in nonlinearity of the $I-V$ curves. The inset plots in Figure 7 show the conductance values over the entire concentration range. 
The only run that led to a change in the degree of rectification (Figure 7c) also yielded a linear increase in the conductance as the DNA concentration was increased. This could be due to physisorption to the pore walls, which would influence the wall surface charge and therefore would influence the bulk charge transfer through the pores. We can speculate that the silanization procedure resulted in a differently charged surface than the other cases, which in turn led to a different adsorption pattern of the ssDNA. However, further tests are required, including an optimization of the silanization process. Plasma-enhanced chemical vapor deposition (PECVD) of silanes may present a more consistent method of producing amino-functionalized surfaces (36).
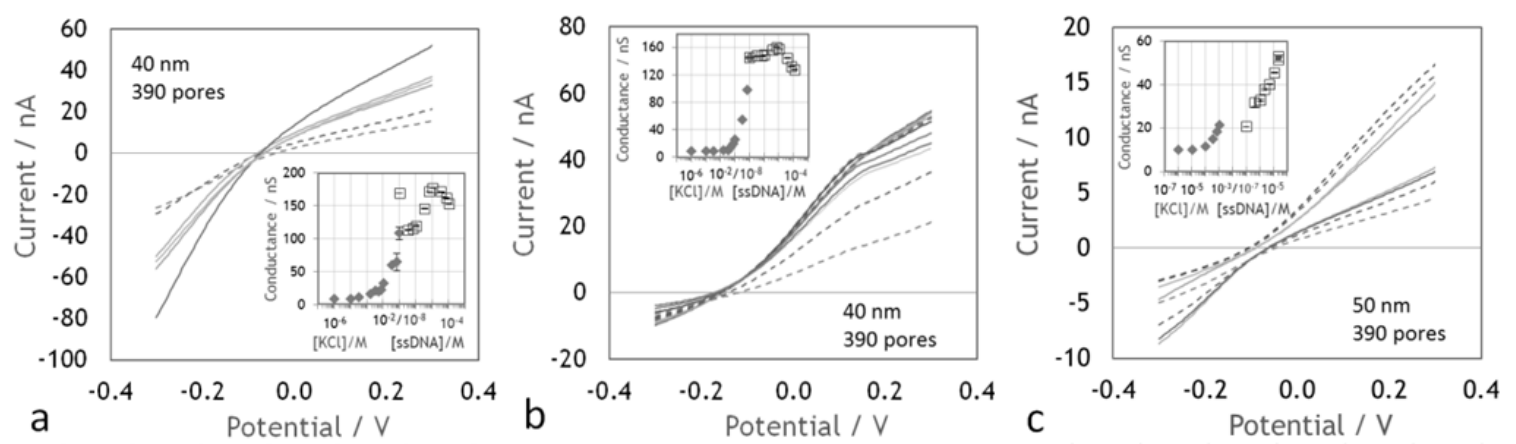

Figure 7. $I$ - $V$ (upper) and conductance (lower) plots for the NA additions method. The $I-V$ curves show the final $\mathrm{KCl}$ additions (dashed lines) and the initial ssDNA (Cap3 with no amino functionalization) additions (solid lines). Only $I-V$ curves just prior to and directly following nucleic acid addition are displayed. The insets show the conductance curves before (closed) and after (open) hybridization. In the insets, the conductance values over the entire concentration range are displayed to demonstrate the curvature. The conductance curve horizontal axis shows the $\mathrm{KCl}$ (left-most) and ssDNA (right-most) concentrations added. Error bars are one standard deviation.

Comparing the results from the offline overnight experiments (Figure 6) and the NA physisorption studies (Figure 7), it is evident that there is some degree of current rectification present in many of the nanopores. This could be due to variety of factors, most notably asymmetric charge distributions along the walls of the pore and asymmetric pore geometry, particularly conically shaped pores. In essence, the current magnitude at the negative end of the potential sweep is different than that for the same potential on the positive side of the sweep. The effect is related to the difference in resistance for ions entering at one end of the pore compared to the other end, either due to geometric or surface charge differences. Conical nanopores may have been formed due the etching process, although this has not been verified through imaging for the membranes used here.

There was also a change in the degree of rectification in one particular case (Figure $7 d$ ). In this, the $I-V$ curve shifts from curved downwards (dashed lines) to curved upwards (solid lines) following hybridization/addition of the nucleic acid. The ssDNA additions that led to such a change (Figure 7d) should not have led to drastic surface charge modifications. Further investigation is required, including optimization of the silanization process, to determine if this is a hybridization event or non-specific adsorption. 
Online Overnight Hybridization. The effects of hybridization over time on current rectification were explored. A set of experiments was performed where the conductance was measured hourly before addition of ssDNA to the functionalized nanopore membrane, and then throughout the overnight hybridization, over 16 hours. Hybridization was measured during this process as the degree of rectification $(4,11)$, which is the ratio of the current at a negative potential versus that at the same positive potential, $r=\left|\mathrm{I}_{-\phi}\right| / \mathrm{I}_{+\phi} \mid$. Preliminary results of current rectification are shown in Figure 8 for complementary, non-complementary, and no DNA added. In Figure 8a, the $I-V$ curves before (dashed) and following DNA addition are shown. In Figure 8b, the degree of rectification is shown for complementary, non-complementary, and no added ssDNA. Each result is normalized to the initial value. It is important to note that, as this is a preliminary study, a variety of pore diameters and numbers were used for the experiments. Also, as in Figures 6 and 7, only $I-V$ curves just prior to and following addition of the ssDNA are presented in Figure 8a and b. The curves at higher and lower concentrations are omitted to facilitate display of the change in nonlinearity of the $I-V$ curves. The curves in Figure 8c show rectification values over the entire experiment.
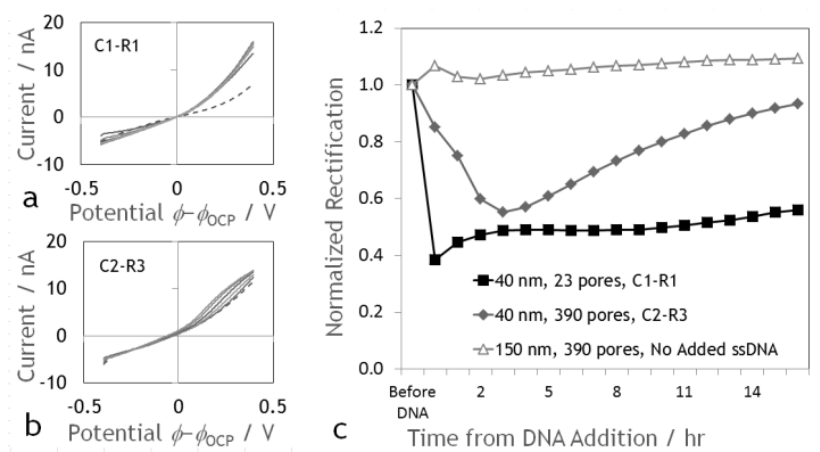

Figure 8. Rectification experiments. $I-V$ curves immediately before and then every hour following addition of (a) complementary ssDNA for to 40-nm, 23-pore array and (b) noncomplementary ssDNA to a $40-\mathrm{nm}, 390$ pore array. For both (a) and (b), only the $I-V$ curves before and immediately after ssDNA addition are shown, and the potential is offset by the open-circuit potential (OCP). (c) Degree of rectification for complementary (black), non-complementary (dark grey), and no added DNA (light grey). The results are normalized to the initial rectification value.

The complementary DNA pair (black squares) show a rapid change in the rectification degree following addition of the ssDNA. Over the course of the 16-hour experiment, the value remained relatively constant. On the other hand, the noncomplementary DNA pair membrane (blue diamond) shows a much slower response in terms of the degree of rectification, over a period of a few hours, then a gradual return to near-baseline over the rest of the experiment. Finally, the control demonstrated little rectification and minimal variation over the course of the run. Since the control experiment was performed with larger pores $(150 \mathrm{~nm})$, it is possible that rectification could not occur, so the lack of signal may solely be due to fluidic effects. Further tests are required to verify this.

It is important to note that these are preliminary results, and require further elucidation of the effects of channel and array geometries, surface charge, and 
hybridization on the rectification behavior. As well, it would be of interest to explore changes in rectification at shorter time intervals directly following hybridization events, as opposed to the one-hour intervals used here. Quicker detection methods for conductance and rectification would allow for real-time hybridization monitoring, and may be able to measure kinetic parameters for such biochemical events. However, this initial study of online rectification demonstrates the promise of this technique for the detection of hybridization using thin nanopore membranes.

\section{Conclusions}

In this study, we have characterized nanopore arrays using conductance measurements, and found that experimental conductance is in qualitative agreement with cylindrical pore model with charged surface. However, this agreement does not match quantitatively, which could be due to conical, as opposed to cylindrical, pores; current rectification; or blocked pores. As well, we applied a surface modification method for attachment of DNA to silicon nitride nanopore membranes. Furthermore, we have observed DNA binding to the surface of modified pore membranes, as detected by changes in current rectification behavior. Using this technique, the rectification signal changed in response to binding of complementary DNA, as compared to noncomplementary DNA, which, again, is consistent with conical pores.

\section{Acknowledgments}

The authors would like to thank Ms. E. Hurley and Mr. J. O'Brien at Tyndall National Institute (Cork, Ireland) for their help throughout this project. The authors are also grateful to Dr. N. Petkov from the Electron Microscopy and Analysis Facility at Tyndall for the SEM imaging. As well, discussions concerning surface modification with Dr. M. Manning (Tyndall National Institute) were invaluable. The authors are grateful to Science Foundation Ireland (grant number 07/IN.1/B967), the Science Foundation Ireland-funded National Access Program (NAP203) and the Irish Research Council for Science, Engineering and Technology (Government of Ireland Postdoctoral Fellowship to JSE) for support of this research. Facilities support provided by the PRTLI4 INSPIRE scheme (Higher Education Authority Ireland).

\section{References}

1. M. Ayub, A. Ivanov, E. Instuli, M. Cecchini, G. Chansin, C. McGilvery, J. Hong, G. Baldwin, D. McComb, J. B. Edel and T. Albrecht, Electrochim. Acta, 55, 8237 (2010).

2. H. S. White and A. Bund, Langmuir, 24, 2212 (2008).

3. Z. Siwy, I. D. Kosińska, A. Fuliński and C. R. Martin, Phys. Rev. Lett., 94, 48102 (2005).

4. I. Vlassiouk, T. R. Kozel and Z. S. Siwy, J. Amer. Chem. Soc., 131, 8211 (2009).

5. S. Datta, A. T. Conlisk, D. M. Kanani, A. L. Zydney, W. H. Fissell and S. Roy, J. Coll. Inter. Sci., 348, 85 (2010). 
6. C. Ho, R. Qiao, J. B. Heng, A. Chatterjee, R. J. Timp, N. R. Aluru and G. Timp, Proc. Natl. Acad. Sci. U.S.A., 102, 10445 (2005).

7. B. Zhang, M. Wood and H. Lee, Anal. Chem., 81, 5541 (2009).

8. R. M. M. Smeets, U. F. Keyser, D. Krapf, M. Y. Wu, N. H. Dekker and C. Dekker, Nano Lett., 6, 89 (2006).

9. D. Stein, M. Kruithof and C. Dekker, Phys. Rev. Lett., 93, 35901 (2004).

10. R. B. Schoch and P. Renaud, Appl. Phys. Lett., 86, 253111 (2009).

11. Y. Choi, L. A. Baker, H. Hillebrenner and C. R. Martin, Phys. Chem. Chem. Phys., 8, 4976 (2006).

12. I. Vlassiouk, P. Takmakov and S. Smirnov, Langmuir, 21, 4776 (2005).

13. X. Wang and S. Smirnov, ACS Nano, 3, 1004 (2009).

14. D. Fologea, M. Gershow, B. Ledden, D. S. McNabb, J. A. Golovchenko and J. Li, Nano Lett., 5, 1905 (2005).

15. S. M. Iqbal, D. Akin and R. Bashir, Nat. Nano, 2, 243 (2007).

16. H. Chang, F. Kosari, G. Andreadakis, M. A. Alam, G. Vasmatzis and R. Bashir, Nano Lett., 4, 1551 (2004).

17. R. Fan, R. Karnik, M. Yue, D. Li, A. Majumdar and P. Yang, Nano Lett., 5, 1633 (2005).

18. O. A. Saleh and L. L. Sohn, Nano Lett., 3, 37 (2003).

19. J. L. Li, M. Gershow, D. Stein, E. Brandin and J. A. Golovchenko, Nat. Mater., 2, 611 (2003).

20. Y. L. Liu and S. M. Iqbal, Appl. Phys. Lett., 95, (2009).

21. Y. Ai, J. Liu, B. Zhang and S. Qian, Anal. Chem., 82, 8217 (2010).

22. K. Luo, T. Ala-Nissila, S. Ying and A. Bhattacharya, Phys. Rev. E, 78, 061911 (2008).

23. M. D. Scanlon, J. Strutwolf, A. Blake, D. Iacopino, A. J. Quinn and D. W. M. Arrigan, Anal. Chem., 1157 (2010).

24. B. Glynn, O. Scheler, S. Parkel, A. Kurg, T. Barry, T. Smith and M. Maher, in IEEE Sensors Conference/2008, 1072 (2008).

25. O. Scheler, B. Glynn, S. Parkel, P. Palta, K. Toome, L. Kaplinski, M. Remm, M. Maher and A. Kurg, BMC Biotechnol., 9, 45 (2009).

26. M. Manning and G. Redmond, Langmuir, 21, 395 (2005).

27. E. B. Kalman, O. Sudre, I. Vlassiouk and Z. S. Siwy, Anal. Bioanal. Chem., 394, 413 (2009).

28. J. Y. Jung, P. Joshi, L. Petrossian, T. J. Thornton and J. D. Posner, Anal. Chem., 81, 3128 (2009).

29. R. Karnik, C. Duan, K. Castelino, H. Daiguji and A. Majumdar, Nano Lett., 7, 547 (2007).

30. Z. S. Siwy, Adv. Func. Mater., 16, 735 (2006).

31. C. Wei, A. J. Bard and S. W. Feldberg, Anal. Chem., 69, 4627 (1997).

32. J. Cervera, B. Schiedt and P. Ramirez, Europhys. Lett., 71, 35 (2005).

33. E. R. Cruz-Chu, A. Aksimentiev and K. Schulten, J. Phys. Chem. C, 113, 1850 (2009).

34. H. Daiguji, Y. Oka and K. Shirono, Nano Lett, 5, 2274 (2005).

35. I. D. Kosinska, I. Goychuk, M. Kostur, G. Schmid and P. Hänggi, Phys. Rev. E, 77, 31131 (2008). 
36. C. Volcke, R. P. Gandhiraman, V. Gubala, J. Raj, T. Cummins, G. Fonder, R. I. Nooney, Z. Mekhalif, G. Herzog, S. Daniels, D. W. M. Arrigan, A. A. Cafolla and D. E. Williams, Biosens. Bioelectron., 25, 1875 (2010). 